\title{
The lateral femoral notch sign: a reliable diagnostic measurement in acute anterior cruciate ligament injury
}

\author{
Prabath C. A. M. Lodewijks ${ }^{1,2}$ - Diyar Delawi ${ }^{1} \cdot$ Thomas L. Bollen $^{3} \cdot$ Gawein R. Dijkhuis $^{3} \cdot$ Nienke Wolterbeek $^{1}$. \\ Jacco A. C. Zijl ${ }^{1}$
}

Received: 26 March 2018 / Accepted: 9 October 2018 / Published online: 13 October 2018

(c) The Author(s) 2018

\begin{abstract}
Purpose To describe the validity and inter- and intra-observer reliability of the lateral femoral notch sign (LFNS) as measured on conventional radiographs for diagnosing acute anterior cruciate ligament (ACL) injury.

Methods Patients ( $\leq 45$ years) with a traumatic knee injury who underwent knee arthroscopy and had preoperative radiographs were retrospectively screened for this case-control study. Included patients were assigned to the ACL injury group $(n=65)$ or the control group $(n=53)$ based on the arthroscopic findings. All radiographs were evaluated for the presence, depth and location of the LFNS by four physicians who were blind to the conditions. To calculate intra-observer reliability, each observer re-assessed $25 \%$ of the radiographs at a 4-week interval.

Results The depth of the LFNS was significantly greater in ACL-injured patients than in controls [median $0.8 \mathrm{~mm}(0-3.1 \mathrm{~mm})$ versus $0.0 \mathrm{~mm}(0-1.4 \mathrm{~mm})$, respectively; $p=0.008$ ]. The inter- and intra-observer reliabilities of the LFNS depth were 0.93 and 0.96, respectively. Secondary knee pathology (i.e., lateral meniscal injury) in ACL-injured patients was correlated with a deeper LFNS [median $1.1 \mathrm{~mm}(0-2.6 \mathrm{~mm})$ versus $0.6 \mathrm{~mm}(0-3.1 \mathrm{~mm}), p=0.012$ ]. Using a cut-off value of $1 \mathrm{~mm}$ for the LFNS depth, a positive predictive value of $96 \%$ was found.

Conclusion This was the first study to investigate the inter- and intra-observer agreement of the depth and location of the LFNS. The depth of the LFNS had a very high predictive value for ACL-injured patients and could be used in the emergency department without any additional cost. A depth of $>1.0 \mathrm{~mm}$ was a good predictor for ACL injury. Measuring the depth of the LFNS is a simple and clinically relevant tool for diagnosing ACL injury in the acute setting and should be used by clinicians in patients with acute knee trauma.
\end{abstract}

Level of evidence Diagnostic study, level II.

Keywords Knee $\cdot$ ACL $\cdot$ General sports trauma $\cdot$ Imaging $\cdot$ Radiology

\section{Introduction}

Prabath C. A. M. Lodewijks

plodewij@umcutrecht.nl

$\triangle$ Jacco A. C. Zij1

J.zijl@ antoniusziekenhuis.nl

1 Department of Orthopaedic Surgery, St. Antonius Hospital, Koekoekslaan 1, 3435 CM Nieuwegein, The Netherlands

2 Department of Rehabilitation, Physical Therapy Science and Sports, University Medical Center Utrecht, Heidelberglaan 100, 3584 CX Utrecht, The Netherlands

3 Department of Radiology, St. Antonius Hospital, Koekoekslaan 1, 3435 CM Nieuwegein, The Netherlands
Diagnosis of anterior cruciate ligament (ACL) injury is challenging in the acute setting. Swelling and pain can lower the reliability of clinical examination [7, 14], and MRI is often not directly available. Misdiagnoses could lead to delays in treatment that may further damage the knee joint. The lateral femoral notch sign (LFNS) has been described as a sign of chronically ACL-deficient knees on conventional radiographs. In chronic injuries, it is defined as a depression more than $1.5 \mathrm{~mm}$ deep in the lateral femoral condyle, near the terminal sulcus $[4,5,10,12,18,19]$. In addition to the depth of the LFNS, the location might also be important for diagnosing ACL injury [5]. The origin of the LFNS was previously thought to be caused by 
repeated pivoting trauma due to chronic instability. More recent studies have described the presence of the LFNS in the acute setting $[4,5,9,13,16,18]$. Nevertheless, the prevalence of a deep LFNS in the acute setting and the reliability of its measurement are not known. Furthermore, there is no clear cut-off point for depth of the impression that is associated with ACL ruptures in the acute setting. Several studies have used various cut-off points $[4,5,9$, $10,16,18,23]$. Due to the simplicity of the measurement, it could be a useful tool for the diagnosis of acute ACL injury, since conventional radiographs are often obtained in the acute setting to rule-out fractures. However, a clear definition regarding the depth of the LFNS with its associated prevalence in patients with acute ACL injuries needs to be obtained first. Furthermore, the reliability of the measurement needs to be evaluated. In this paper, the LFNS was evaluated as a valuable diagnostic tool for ACL injury in the acute setting by assessing the predictive value and the inter- and intra-observer reliability.

The purpose of this study was to describe the validity and reliability of the LFNS depth measurement in conventional radiographs. It was hypothesized that this measurement could be of additional value in diagnosing ACL injury in the acute setting.

\section{Materials and methods}

In this case-control study, all patients with traumatic knee injury who underwent knee arthroscopy at our institution between May and December 2013 were evaluated. The inclusion criteria were that patients must be between the age of 12 and 45 years and that preoperative radiographs must be available. The exclusion criteria were: known previous knee fractures, previous ACL rupture, osteoarthritis, or a delay of more than 4 months between injury and imaging (to exclude chronic ACL injuries). The patients included in the study were assigned to the ACL injury group or the control group (no ACL injury), depending on arthroscopic findings. All radiographs were evaluated for the presence, depth and location of the lateral femoral notch sign by 4 blinded physicians that were involved in the diagnosis of the ACL injuries. Blinding was accomplished using a digital PACS viewer; the physicians were only able to see the relevant anonymous radiographs and had no access to additional patient information or medical history. Furthermore, retrospectively, information regarding the arthroscopy, including the presence of chondral defects, meniscal injuries and ligament ruptures, were collected from patient files. Baseline characteristics including gender, age, sport, and clinical examination were also retrieved.

\section{Radiological measurements}

All lateral radiographs of the knees were made using digital $\mathrm{X}$-ray machines under standard operating procedures, resulting in radiographs with equal magnification factors. The LFNS was measured using a digital PACS viewer (Fig. 1). A tangent line was drawn from the normal articular surface of the lateral femoral condyle. A line from the tangent line to the deepest point of the lateral femoral notch gave the depth of the LFNS $[1,9,10]$. Based on the literature, the LFNS was divided into four categories, namely depths of $0.0 \mathrm{~mm}$, 0.1-1.0 mm, 1.1-1.4 mm, and $\geq 1.5 \mathrm{~mm} \mathrm{[4,} \mathrm{5,} \mathrm{9,} \mathrm{10,} \mathrm{16,} \mathrm{18,}$ 23]. The first two categories were considered normal depths, and the last 2 were considered pathological categories. To determine the location of the LFNS, the Blumensaat line was used $[8,11]$. The Blumensaat line is a line through the roof of the intercondylar notch of the femur seen on the lateral radiograph of the knee. To determine the exact location, a tangent line to the lateral femoral condyle was drawn from the posterior beginning of the LFNS to the Blumensaat line [8]. A normal terminal sulcus does not extend more than $10 \mathrm{~mm}$ posterior to the Blumensaat line [5]. The location between the LFNS and the Blumensaat line was divided into three categories: $0 \mathrm{~mm}, 1-10 \mathrm{~mm}$ and $>10 \mathrm{~mm}$ (Fig. 2) [18]. These categories were chosen, based on the literature, to differentiate between normal and pathological locations $[5,8]$. In this study, the depth and the location of the LFNS were combined to determine if a pooled measurement resulted in a more accurate prediction of ACL injury than isolated measurements of depth and location. Furthermore, the incidence of lateral meniscal injury and its correlation with the depth of the LFNS was studied $[3,9,10,13,16,27]$.

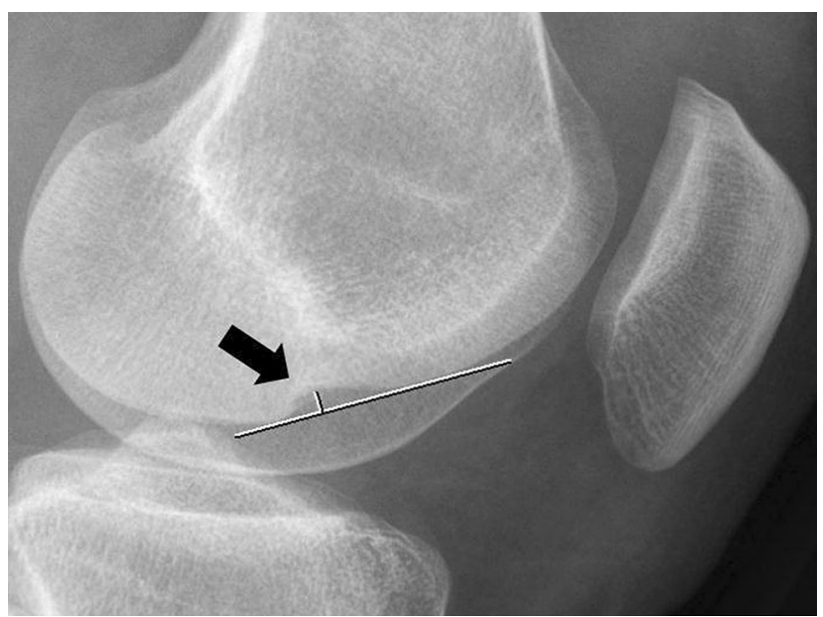

Fig. 1 ACL-deficient knee with a deep lateral femoral notch sign. The depth was measured by drawing a line over the femoral condyle (long line) and a small line perpendicular to this line and the deepest point of the depression (black arrow) 


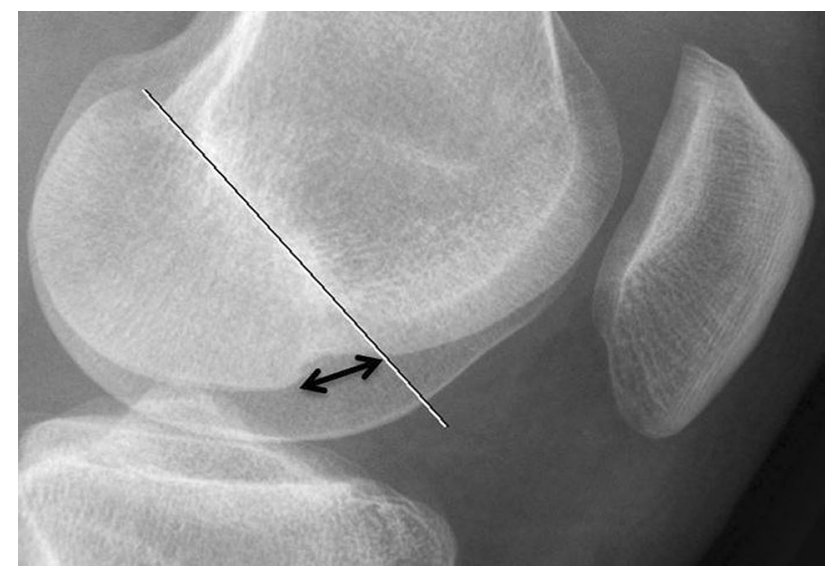

Fig. 2 ACL-deficient knee illustrating the measured location for the lateral femoral notch sign (LFNS). The white line is the Blumensaat line. The black double-headed arrow (tangent line) is the distance between the Blumensaat line and the posterior beginning of the LFNS

\section{Inter- and intra-observer reliability}

All radiographs were evaluated by four specialists, consisting of two radiologists, an orthopaedic surgeon and a sports medicine physician. To calculate intra-observer reliability, each observer re-assessed $25 \%$ of the radiographs at a 4 -week interval to prevent recall bias. The observers measured the depth of the LFNS within tenths of a mm and the location of the LFNS using its location relative to Blumensaat line in $\mathrm{mm}$. All assessors were blinded to the characteristics and diagnoses of the patients and had no information about the arthroscopy. The radiographs were assessed on a high-contrast computer screen with a picture archive and communication system viewer. This retrospective study did not have to be reviewed by a medical ethics board according to the Dutch Law on Medical Research with Humans (WMO).

\section{Statistical analysis}

Statistical analyses were performed using the statistical program SPSS ${ }^{\circledR}$ (Statistical Package for the Social Sciences, Chicago, IL, Version 22.0). For differences between ACLinjured and non-ACL-injured knees, the Mann-Whitney $U$ test was used for continuous data, and Fisher's exact test was used for categorical data. According to general recommendations for evaluating measurement properties, at least 50 patients needed to be included [24]. Therefore, the aim was to include at least 100 patients.

Inter- and intra-observer agreement was measured using the intraclass correlation coefficient (ICC) with a two-way random effects model, single measurements and absolute agreement [22]. In general, an ICC of 0.70 is recommended as a minimum standard for test-retest reliability; an ICC of less than 0.40 is described as weak, between 0.41 and 0.60 is described as moderate, between 0.61 and 0.80 is described as substantial and $>0.81$ is described as almost perfect agreement [25]. The $95 \%$ confidence interval (CI), the range of values containing the $95 \%$ confidence (the "true" correlation coefficient), was also calculated for the ICC.

Correlations in the ACL-injured group were measured with Spearman's rho. Logistic regression analysis was used to find associations between the depth of the LFNS and other intra-articular injury, such as meniscal tears and chondral defects.

\section{Results}

118 patients were eligible for this study, including 65 patients with ACL injury and 53 patients without ACL injury (control group) (Table 1). The ACL-injured group was younger, had more sport-related trauma and had a lower percentage of medial meniscal injuries. The sport-related trauma was mostly in high-risk pivoting sports (i.e., soccer, tennis, and basketball) [9].

All patients underwent arthroscopy. In the ACL-injured group, a reconstruction of the ACL was performed. Most of the control patients underwent arthroscopy due to a medial or lateral meniscal tear (67.9\% and $22.6 \%$, respectively). The remaining $10 \%$ underwent arthroscopy because of chondral lesions.

\section{Radiological assessment}

Four observers assessed all 118 radiographs and measured the depth and location of the LFNS. The median LFNS depth was $0.8 \mathrm{~mm}$ in ACL-injured patients and $0.0 \mathrm{~mm}$ in the control group $(p=0.008)$ (Table 1). The median LFNS location in relation to the Blumensaat line was $9 \mathrm{~mm}$ in ACL-injured patients and $0 \mathrm{~mm}$ in the controls $(p<0.001)$ (Table 1). The distribution of scores within the various categories for the depth and location of the LFNS was significantly different between the ACL-injured patients and the controls (both $p<0.001$ ). The positive and negative predictive values (PPV and NPV, respectively) of the various cutoffs and the combined LFNS location and depth values are shown in Table 2.

\section{Inter- and intra-observer agreement}

The inter-observer values for the four observers were in almost perfect agreement for the LFNS depth (0.93) and were in substantial agreement for the location (0.64). The intra-observer values for depth and location were in almost perfect agreement (0.96) and moderate agreement (0.59), respectively. The combination of sulcus location and 
LFNS depth was also tested for inter- and intra-observer agreement (Table 3).

Table 1 Patient characteristics and the location and depth measurements of the LFNS $(\mathrm{mm})$

Table 2 Positive predictive value (PPV), negative predictive value (NPV), sensitivity and specificity for different cut-off points

Table 3 Inter- and intraobserver values of the LFNS depth and location between the sulcus and Blumensaat line

$S D$ standard deviation

\section{Correlations and associations}

In the ACL-injured group, 26 patients (40\%) also had a lateral meniscal injury. The median LFNS depth of patients

\begin{tabular}{|c|c|c|c|}
\hline & ACL injury $(n=65)$ & Controls $(n=53)$ & $p$ value \\
\hline Male gender (\%) & $43(66.2 \%)$ & $32(60.4 \%)$ & n.s. \\
\hline Mean age in years (SD) & $25.4( \pm 6.7)$ & $32.5( \pm 9.2)$ & $<0.001$ \\
\hline Sport trauma & $57(87.7 \%)$ & $9(17.0 \%)$ & $<0.001$ \\
\hline Football & $30(46.2 \%)$ & $3(5.7 \%)$ & \\
\hline Hockey & $9(13.8 \%)$ & $3(5.7 \%)$ & \\
\hline Tennis & $1(1.5 \%)$ & $1(1.9 \%)$ & \\
\hline Other & $17(26.2 \%)$ & $2(3.8 \%)$ & \\
\hline Medial meniscal injury & $15(23.1 \%)$ & $36(67.9 \%)$ & $<0.001$ \\
\hline Lateral meniscal injury & $26(40.0 \%)$ & $12(22.6 \%)$ & n.s \\
\hline \multicolumn{4}{|l|}{ Depth of LFNS } \\
\hline Median (range) & $0.8 \mathrm{~mm}(0-3.1 \mathrm{~mm})$ & $0.0 \mathrm{~mm}(0-1.4 \mathrm{~mm})$ & 0.008 \\
\hline \multicolumn{4}{|l|}{ Category } \\
\hline $0.0 \mathrm{~mm}$ & $9(13.8 \%)$ & $27(50.9 \%)$ & $<0.001$ \\
\hline $0.1-1.0 \mathrm{~mm}$ & $32(49.2 \%)$ & $25(47.2 \%)$ & \\
\hline $1.1-1.4 \mathrm{~mm}$ & $14(21.5 \%)$ & $1(1.9 \%)$ & \\
\hline$\geq 1.5 \mathrm{~mm}$ & $10(15.4 \%)$ & $0(0 \%)$ & \\
\hline \multicolumn{4}{|l|}{ Location } \\
\hline Median (range) & $9 \mathrm{~mm}(0-19 \mathrm{~mm})$ & $0 \mathrm{~mm}(0-17 \mathrm{~mm})$ & $<0.001$ \\
\hline \multicolumn{4}{|l|}{ Category } \\
\hline $0 \mathrm{~mm}$ & $14(21.5 \%)$ & $30(56.6 \%)$ & $<0.001$ \\
\hline $1-10 \mathrm{~mm}$ & $24(36.9 \%)$ & $17(32.1 \%)$ & \\
\hline$>10 \mathrm{~mm}$ & $27(41.5 \%)$ & $6(11.3 \%)$ & \\
\hline
\end{tabular}

\begin{tabular}{lllll}
\hline Cut-off values & PPV (\%) & NPV (\%) & Sensitivity (\%) & Specificity (\%) \\
\hline LFNS $\geq 1.5 \mathrm{~mm}$ & 100 & 49.1 & 15.4 & 100 \\
LFNS $>1.0 \mathrm{~mm}$ & 96.0 & 55.9 & 36.9 & 98.1 \\
Location $>10 \mathrm{~mm}$ & 81.8 & 55.3 & 41.5 & 88.7 \\
Combination LFNS $>1.0 \mathrm{~mm}$ AND & 100 & 53.0 & 27.7 & 100 \\
$\quad$ location $>10 \mathrm{~mm}$ OR LFNS $\geq 1.5 \mathrm{~mm}$ & & & & 86.8 \\
LFNS $>1.0$ OR location $>10 \mathrm{~mm}$ & 83.3 & 60.5 & 53.9 & \\
\hline
\end{tabular}

\begin{tabular}{lllrr}
\hline Measurement in mm & Single measures & $95 \%$ CI & $F(d f 1, d f 2)$ & $p$ value \\
\hline $\begin{array}{l}\text { Inter-observer agreement (ICC) } \\
\quad\end{array}$ & 0.93 & & & \\
$\quad$ LFNS depth & 0.64 & $0.91-0.95$ & $54.81(117,351)$ & $<0.001$ \\
$\quad$ Location & 0.80 & $0.55-0.72$ & $8.64(116,348)$ & $<0.001$ \\
Combination of location and depth & $0.75-0.85$ & $17.54(117,351)$ & $<0.001$ \\
Intra-observer agreement (ICC) & & & & \\
$\quad$ LFNS Depth & 0.96 & $0.94-0.97$ & $48.36(117,117)$ & $<0.001$ \\
Location & 0.59 & $0.45-0.70$ & $3.98(117,117)$ & $<0.001$ \\
Combination of location and depth & 0.84 & $0.78-0.89$ & $11.66(117,117)$ & $<0.001$ \\
\hline
\end{tabular}


in the ACL-injured group with lateral meniscal injury was $1.1 \mathrm{~mm}$ versus $0.6 \mathrm{~mm}$ in patients without lateral meniscal injury $(p=0.012) .80 \%$ of the 10 ACL-injured patients with an LFNS depth of $\geq 1.5 \mathrm{~mm}$ had a lateral meniscal tear compared to only $33 \%$ of patients (18 of 55 patients) with an LFNS depth $<1.5 \mathrm{~mm}$, resulting in an odds ratio of 8.2 $(p=0.011,95 \%$ CI 1.6-42.7). In the control group, there was no difference in LFNS depth between patients with or without lateral meniscal injury.

In the total population (ACL-injured and controls), there were 38 lateral meniscal injuries (26 in ACL-injured patients, 12 in controls), and the median LFNS depth in lateral meniscal injury was $0.8 \mathrm{~mm}$ compared to $0.5 \mathrm{~mm}$ in the absence of lateral meniscal injury $(p=0.040)$ (Fig. 3).

\section{Discussion}

The most important finding of the present study was that the LFNS is a clinically relevant tool for diagnosing acute ACL injuries. Furthermore, the inter-and intra-observer values showed that the depth and location of the LFNS could be reliably measured. It was postulated that a cutoff value of $>1 \mathrm{~mm}$ should be used in patients with acute knee injuries. When the depth of the impression of the LFNS was greater than $1 \mathrm{~mm}$, an excellent positive predictive value (96\%) and an acceptable negative predictive value $(56 \%)$ were found. Using a higher cut-off value of $1.5 \mathrm{~mm}$ did not significantly increase the PPV or the NPV. However, a higher cut-off value had a substantial negative

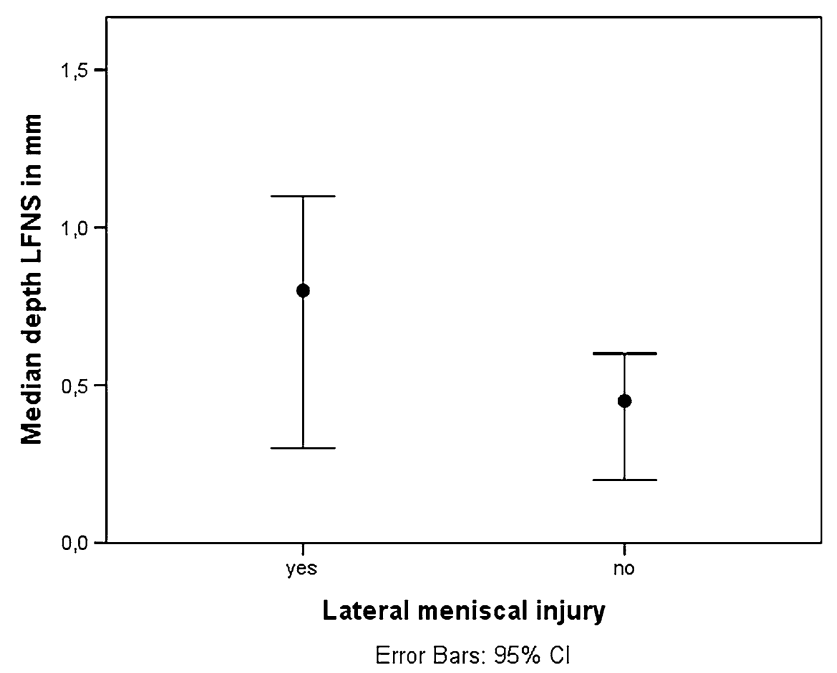

Fig. 3 The left side shows the median lateral femoral notch sign depth in patients with lateral meniscal injury $(n=38)$, with a median depth of $0.8 \mathrm{~mm}$; the right side shows the median lateral femoral notch sign depth in patients without lateral meniscal injury $(n=80)$, with a median depth of $0.5 \mathrm{~mm}(p=0.040)$. The vertical lines show the $95 \% \mathrm{CI}$ effect on the sensitivity of the test, decreasing it from 37 to $15 \%$. The cut-off values found in this study were lower compared to those of other studies in (chronic) ACL-deficient knees [4, 9, 10, 18, 20,26]. This could be explained by chronic instability that could result in repetitive trauma to the lateral femoral condyle and, subsequently, increased LFNS depth. The location of the LFNS, whether or not it was combined with the depth of the impression, did not contribute to the diagnostic value of the test.

The reliability of the LFNS depth measurement was very high. This was shown by high inter- and intraobserver reliability scores (ICCs of 0.93 and 0.96 , respectively) [25]. The location measurement was less reproducible, with ICCs of 0.64 and 0.53 for inter- and intra-observer reliability, respectively. One of the researchers showed larger variations than the others, possibly due to inexperience in performing radiological measurements. For this reason, we recommend the sole use of the LFNS depth to predict the occurrence of ACL rupture in acute knee trauma.

In addition to diagnosing ACL injury using the LFNS depth, secondary knee pathology is more likely with a deep LFNS. Patients with lateral meniscal injury in combination with ACL injury had a significantly deeper LFNS than those without lateral meniscal injury $[9,10,13]$. This could be explained by the more forceful mechanism of trauma and impingement of the meniscus between the lateral femoral condyle and the tibial plateau.

There are studies describing the reliability of clinical examination (Lachman, anterior drawer, and pivot shift) in ACL-injured knees. Sensitivity and specificity were $0.63-0.93$ and $0.55-0.99$, respectively, for the Lachman test, which was described as the most reliable test in most studies [2, 14, 15, 17, 21]. Most studies did not describe clinical examination in cases of ACL injury in the acute setting. One small study found a low number of recognized ACL ruptures in the emergency department; of 27 ACL ruptures, only seven were diagnosed [7,14]. Although this was a small study, it suggested that clinical examination was less reliable in an acute setting. A study by Katz (1986) showed a lower sensitivity of all ACL tests within 2 weeks of injury, compared to after 2 weeks [14]. The sensitivity and specificity of the LFNS in this study was comparable to those of clinical tests in the acute setting.

This study had some limitations. The study group was relatively small; nevertheless, significant differences between ACL-injured patients and controls were found. The results showed that the Blumensaat line had larger inter- and intraobserver variation [11]. Observers reported that it was often difficult to determine the beginning of the terminal sulcus. This was mostly seen in the less-experienced observer and had an effect on the inter- and intra-observer agreement of the measurement. 


\section{Conclusion}

This was the first study to investigate the inter- and intraobserver agreement of the depth and the location of the lateral femoral notch sign. The depth of the lateral femoral notch sign had a very high predictive value in ACL-injured patients and could be used in the emergency department without any additional cost. A depth of $>1.0 \mathrm{~mm}$ was a good predictor of ACL injury. Measuring the depth of the LFNS is a simple and clinically relevant predictor for diagnosing ACL injury in the acute setting and should be used by clinicians in patients with acute knee trauma.

Funding None.

\section{Compliance with ethical standards}

Conflict of interest All authors declare that they have no conflict of interest.

Ethical aproval This retrospective study did not have to be reviewed by a medical ethics board according to the Dutch Law on Medical Research with Humans (WMO).

Open Access This article is distributed under the terms of the Creative Commons Attribution 4.0 International License (http://creativeco mmons.org/licenses/by/4.0/), which permits unrestricted use, distribution, and reproduction in any medium, provided you give appropriate credit to the original author(s) and the source, provide a link to the Creative Commons license, and indicate if changes were made.

\section{References}

1. Bell DJ, Chia-Tsong Hu et al (2013) Lateral femoral notch sign (knee). https://radiopaedia.org/articles/lateral-femoral-notch-signknee. Accessed 18 Jan 2018

2. Benjaminse A, Gokeler A, van der Schans CP (2006) Clinical diagnosis of an anterior cruciate ligament rupture: a meta-analysis. J Orthop Sports Phys Ther 36:267-288

3. Bisson LJ, Kluczynski MA, Hagstrom LS et al (2013) A prospective study of the association between bone contusion and intraarticular injuries associated with acute anterior cruciate ligament tear. Am J Sports Med 41:1801-1807

4. Burgers PTPW, Hollander PHC (2016) A typical sign on a plain knee radiograph. BMJ 355:i6021

5. Garth WP Jr, Greco J, House MA (2000) The lateral notch sign associated with acute anterior cruciate ligament disruption. Am J Sports Med 28:68-73

6. Grimberg A, Shirazian H, Torshizy H et al (2015) Deep lateral notch sign and double notch sign in complete tears of the anterior cruciate ligamentL MR imaging evaluation. Skeletal radiol 44:385-391

7. Guillodo Y, Rannou N, Dubrana F et al (2008) Diagnosis of anterior cruciate ligament rupture in an emergency department. J Trauma 65:1078-1082
8. Hacking C, Goeld A et al (2013) Blumensaat line. https://radio paedia.org/articles/blumensaat-line-1. Accessed 18 Jan 2018

9. Herbst E, Hoser C, Tecklenburg K et al (2015) The lateral femoral notch sign following ACL injury: frequency, morphology and relation to meniscal injury and sports activity. Knee Surg Sports Traumatol Arthrosc 23:2250-2258

10. Hoffelner T, Pichler I, Moroder P et al (2015) Segmentation of the lateral femoral notch sign with MRI using a new measurement technique. BMC Musculoskelet Disord 16:217

11. Iriuchishima T, Ryu K, Aizawa S et al (2016) Blumensaat's line is not always straight: morphological variation of the lateral wall of the femoral intercondylar notch. Knee surg Sports Traumatol Arthrosc 24:2752-2757

12. Jaremko JL, Guenther ZD, Jans LB et al (2013) Spectrum of injuries associated with paediatric ACL tears: an MRI pictorial review. Insights Imaging 4:273-285

13. Kanakamedala AC, Burnham JM, Pfeiffer TR et al (2018) Lateral femoral notch depth is nog associated with increased rotatory instability in ACL-injured knees: a quantitative pivot shift analysis. Knee Surg Sports Tramatol Arthrosc 26:1399-1405

14. Katz JW, Fingeroth RJ (1986) The diagnostic accuracy of ruptures of the anterior cruciate ligament comparing the Lachman test, the anterior drawer sign, and the pivot shift test in acute and chronic knee injuries. Am J Sports Med 14:88-91

15. Malanga GA, Andrus S, Nadler SF et al (2003) Physical examination of the knee: a review of the original test description and scientific validity of common orthopedic tests. Arch Phys Med Rehabil 84:592-603

16. Nakauchi M, Kurosawa H, Kawakami A (2000) Abnormal lateral notch in knees with anterior cruciate ligament injury. J Orthop Sci 5:92-95

17. Ostrowski JA (2006) Accuracy of 3 diagnostic tests for anterior cruciate ligament tears. J Athl Train 41:120-121

18. Pao DG (2001) The lateral femoral notch sign. Radiology 219:800-801

19. Patel SA, Hageman J, Quatman CE et al (2014) Prevalence and location of bone bruises associated with anterior cruciate ligament injury and implications for mechanism of injury: a systematic review. Sports Med 44:281-293

20. Sanders TG, Folio LR (2007) The deep lateral femoral notch: a sign of anterior cruciate ligament disruption. Mil Med 172:8-17

21. Scholten RJ, Deville WL, Opstelten W et al (2001) The accuracy of physical diagnostic tests for assessing meniscal lesions of the knee: a meta-analysis. J Fam Pract 50:938-944

22. Shrout PE, Fleiss JL (1979) Intraclass correlations: uses in assessing rater reliability. Psychol Bull 86:420-428

23. Speer KP, Spritzer CE, Bassett FH et al (1992) Osseous injury associated with acute tears of the anterior cruciate ligament. Am J Sports Med 20:382-389

24. Terwee CB, Bot SD, de Boer MR et al (2007) Quality criteria were proposed for measurement properties of health status quastionnaires. J Clin Epidemiol 60(1):34-42

25. Viera AJ, Garrett JM (2005) Understanding interobserver agreement: the kappa statistic. Fam Med 37:360-363

26. Yeung KW, Liu GC, Wu DK (1998) Tear of the anterior cruciate ligament: evaluation with MR imaging. Kaohsiung J Med Sci 14:88-93

27. Yoon KH, Yoo JH, Kim KI (2011) Bone contusion and associated meniscal and medial collateral ligament injury in patients with anterior cruciate ligament rupture. J Bone Jt Surg Am 93:1510-1518 